\title{
XX.
}

\section{Über die Bedeutung der Langerhansschen Inseln im menschlichen Pankreas}

(mit besonderer Berücksichtigung der durch Methylgrün-Pyroninfärbung gewonnenen Resultate).

(Aus dem Pathologischen Institut der Universität Berlin.)

Von

Dr. Karl Koch,

Assistenten am Institute.

(Hierzu Taf. VII und 2 Textfiguren.)

Bei Untersuchung des menschlichen Pankreas bediene ich mich seit längerer Zeit, einer Anregung Professor P a p p e $\mathrm{n}$ h e i m s folgend, der M e th y g r ü n P y r o n inf ärbung. Wie so häufig Fortschritte in der histologischen Technik uns neue wissenschaftliche Erkenntnisse erleichtert haben, so glaube ich, daß auch dieAnwendung dieser Färbung b e i d er Un ter s u c h u n g des Pankreas uns manche wertvolle Aufklärung geben kann. - Es sei mir zuerst gestattet, die von mir angewandte $\mathrm{Technik}$ kurz zu skizzieren.

Ein Haupterfordernis für gutes Gelingen der Färbung ist die geeignete Fix i e ru ng des Objektes, und hier versagen bis auf den Alkohol eigentlich alle unseren gebräulichen Fixierungsmittel mehr oder weniger. Besonders die in der pathologischen Hitologie so viel angewandten Fixierungsmittel Formol und Mü $1 \mathrm{l}$ e r - Formol geben nach meinen Erfahrungen ganz unsichere, meist sogar schlechte Resultate. Auch mit dem von $\mathrm{P}$ a $\mathrm{p}$ p e $\mathrm{n} \mathrm{h}$ e i m ${ }^{1}$ ) neuerdings angegebenen „M ü 11 er-Alkohol", einem Gemisch von Alkohol und M üll e rscher Flüssigkeit, habe ich nur schlechte Erfahrungen gemacht, und ich kann nicht finden, daß diese Kombination irgendwelche Vorteile böte. Gewiß hat die Fixierung in reinem Alkohol das Mißliche an sich, daß Schrumpfungen an den Gewebselementen nur schwer völlig zu vermeiden sind, aber es ist nach meinen Erfahrungen für das gute Gelingen der Methylgrün-Pyronin-Färbung auch durchaus nicht erforderlich, daß die Fixierung mit einem Alkohol höherer Konzentration begonnen wird. Mit einem schwächeren als 70 prozentigen Alkohol zu beginnen, scheint allerdings nicht zweckmäßig zu sein.

Als Einbettungsmethode hat sich mir am meisten die Paraffineinbettung bewährt. Abgesehen davon, daß nur bei dieser Methode genügend leicht dünne Schnitte erhältlich sind, schien es mir auch, als ob bei Zelloidinschnitten die Färbung lange nicht so schöne und klare Bilder gäbe. Auch vorherige Entzelloidinierung der Schnitte brachte keine erheblichen Vorteile.

Was die F ärbung selbst anbetrifft, so färbte ich die entparaffinierten Schnitte, gleichgültig ob aufgeklebt oder flottierend, bєi Zimmertemperatur 5 Minuten in dem von $\mathrm{G} r$ ü ble $\mathrm{r}$ bezogenen Farbgemisch. Die Schnitte wurden darauf in destilliertem Wasser so lange abgespült, bis keine größeren Farbwolkon mehr abgingen, mit Fließpapier abgetrocknet und in reinem Azeton ausdifferenziert und entwässert. Darauf Übertragen in Xylol und Einschluß in Kanadabalsam. Nach dieser Methode habe ich gleichmäßig gute Resultate bei Anwendung der Färbung auf die verschiedensten Gewebsteile bekommen ${ }^{\mathrm{I}}$ ).

1) Wie zu erwarten, bekommt man recht schöne Bilder auch von den übrigen Speicheldrüsen bei Methylgrün-Pyroninfärbung. Die ,serösen“ Zellen dieser Drüsen färben sich rot, die

Virchows Archiv f. pathol. Anat. Bd. 211. Hft. 3. 
Die Anwendung dieser Färbung bei der Untersuchung des Pankreas ergibt nun außerordentlich instruktive und schöne Bilder, da sich die Tubuluszellen anders färben wie die Inselzellen, anders wieder die ,zentroazinären" Zellen und die Epithelien der Ausführungsgänge (siehe Taf. VII, Fig. 1a und 1b).

Was zuerst die T u bu l u $\mathrm{s}$ e 11 e $\mathrm{n}$ anbetrifft, so treten sie als leuchtend rot gefärbte Gebilde sehr deutlich hervor. Ihr Protoplasmafarbt sich sehr intensiv m it dem P y r o n in, doch so, daß die Farbe nicht diffus das Protoplasma des Zelleibes durchtränkt, sondern es färben sich kleine, unregelmäBig geformte und, auch bei Betrachtung mit Ölimmersion, nicht deutlich abzugrenzende Körnchen leuchtend rot. Diese äuBerst feinen Körnchen sind im Protoplasma der Zelle nicht gleichmäBig verteilt und sind nicht etwa identisch mit den Zymogenkörnchen. Im Gegenteil, wo letztere vorhanden sind, sieht man helle Lücken, die um ein Vielfaches größer sind als die geschilderten gefärbten Partikelchen. Gerade die Anwesenheit von Zymogenkörnchen scheint die Verschiedenheit in der Färbungsintensität der einzelnen Teile des Zelleibes zu bewirken, denn es sind gerade die zentralen, dem Lumen des Tubulus zugewandten Teile der Zellen, die sich durch mehr rosarote Färbung von dem Dunkelrot der basalen Teile abheben, eine Folge der in den zentralen Teilen geringeren Anzahl gefärbter Körnchen. In einer Anzahl von Schnitten pathologisch veränderter Bauchspeicheldrüsen fehlte dieser Färbungsunterschied. Es lag dann aber auch der Kern sehr viel weiter nach dem Tubuluslumen zu, und helle Fleckchen, die für das Vorhandensein von Zymogenkörnchen sprechen, waren nicht aufzufinden. Es handelte sich in diesen Fällen vielleicht um Zellen im Stadium der paralytischen Sekretion. Die Kerne der Tubuluszellen nehmen, ebenso wie alle anderen Kerne, bei der Methylgrün-Pyroninfärbung eine schöne blaugrüne bis blaugraue Färbung an.

Beträchtlich anders als die Tubuluszellen verhalten sich die $\mathrm{Z}$ ell e $\mathrm{n}$ der Langerhans schen In seln gegenüber dieser Färbung, ein Unterschied, der schon bei Betrachtung der Schnitte mit schwachem Objektiv deutlich in die Augen fällt und die Auffindung der $\mathrm{L}$ a $\mathrm{n} \mathrm{g}$ e $\mathrm{r} \mathrm{h}$ a $\mathrm{n}$ s schen Inseln leichter macht, als bei fast allen anderen Färbungen. Bei schwacher VergröBerung heben sich

schleim-sezernierenden tief blangrïn. Daher treten so die Gi a n u z zi schen „Halbmonde" besonders deutlich hervor. Vorteilhaft ist die Färbung bei der Untersuchung der Leber, bei der sich die Leberzellen sehr gut durch rote Färbung vom übrigen Gewebe, besonders den Gallengangsepithelien abheben. Auch beim Endometrium und der Naserschleimhaut bekommt man hübsche Bilder, da das Zellprotoplasma zahlreiche rotgefärbte Körnehen enthält. In Gallertkrebsen wieder hebt sich der Schleim durch dunkel blaugrüne Farbe gut ab. So mögen noch manche Gewebsarten zweckmäßig durch Anwendung dieser, technisch ja ziemlich einfachen, Färbung untersucht werden. In manchen Fällen fand ich keine Vorteile; so bei Untersuchung von Hoden, Nieren, Thymus und Lymphdrüsen. Eine für das gute Gelingen der Färbung auch hier unerläßliche Vorbedingung ist möglichste Lebensfrische der Organteile. 
nämlich die Zellinseln sehr deutlich von dem übrigen rotgefärbten Parenchym als grünliche Flecken ab, die nur einen ganz schwach rötlichen Farbenton haben. Aber auch bei starker Vergrößerung sind die Färbungsdifferenzen sehr scharf, ein Vorteil gegenüber allen anderen Färbungen (auch der Färbung nach van Gi eson), die in dieser Beziehung, wie Herxhoimer ${ }^{2}$ sehr richtig bemerkt, stark versagen. Bei starker Vergrößerung sieht man, daß die Inselzellen ein im wesentlichen ungefärbtes, nur in Ausnahmefällen ganz schwach rosa tingiertes Protoplasma besitzen. Sehr interessant ist nun, daß der Zelleib in ziemlich wechselnder Menge, meist aber nur vereinzelt, kleine intensiv rot gefärbte Körnchen einschließt, die in jeder Beziehung den im Protoplasma der Tubuluszellen so sehr zahlreichen Körnchen gleichen. Im unveränderten Pankreas gelang es immer, diese Körnchen in den Inselzellen nachzuweisen, anders dagegen in manchen Fällen von schweren Pankreaserkrankungen (mit und ohne Diabetes!), wo diese Gebilde manchmal völlig fehlten und die Zelleiber nur eine schwache diffuse Rosafärbung annahmen. Wie dieser abweichende Befund vielleicht zu erklären ist, möchte ich weiter unten besprechen.

Eine diffuse Hellrosafärbung nehmen nun auch die Epithelien der

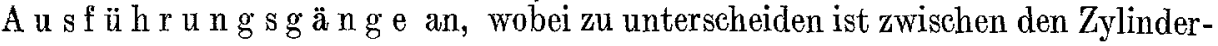
zellen der größeren Gänge und den mehr kubischen der kleinsten. Nur erstere nämlich zeigen die beschriebene Färbung, die anderen haben einen mit Pyronin völlig ungefärbtenZelleib und verhalten sich gerade wie die , z e n t r o a z i n ä r e n “ $\mathrm{Z}$ e 11 e $\mathrm{n}$, die ebenfalls völlig ungefärbtes, beziehungsweise ganz matt grünliches Protoplasma haben.

Kann nun die Anwendung der Methylgrün-Pyroninfärbung uns die Beantwortung der Frage erleichtern: „Sind die Langerhansschen Inseln Gebilde sui generis" oder, "weleher Zusammenhang besteht zwischenden Inselzellen und dem übrigen Parenchym, besonders den Tubuluszellen"? Ich glaube ja, und ich glaube weiter, daß die durch diese Färbung erleichterte und vertiefte Erkenntnis des Baues der Bauchpseicheldrüse die Ansicht der Autoren stützen muß, die wie Herxheimer, v. Hansemann ${ }^{3}$, Karakascheff ${ }^{4}$ u. a. im Gegensatz zu den Forschern, als deren Hauptvertreter wohl W e i c h s e l ba um ${ }^{5}$ zu gelten hat, die Langerhansschen Inseln nicht als selbständige Gebilde gelten lassen wollen und Übergänge zwischen Insel- und Tubuluszellen behaupten. Was mir für die Richtigkeit dieser Anschauung zu sprechen scheint, möchte ich in folgendem ausführen.

Die zahlreichen Beobachtungen sogenannter „Übergangsbilder" zwischen Insel- und Tubuluszellen sind von den Anhängern der Ansicht, daß die L a n g e r h a n s schen Inseln selbständige Gebilde seien, häufig mit dem Einwand zu widerlegen gesucht, $\mathrm{da}$ B $\mathrm{zwar}$ gelegentlich innerhalb von Inseln vereinzelte Tubulus. 
zellen gefunden werden können, daß aber dieser Befund auf einer Zufälligkeit der Schnittrichtung beruhe und die, von dieser Seite als sicher vorhanden angenommene, Kapsel trennend zwischen den hellen Inseln- und den granulierten Tubuluszellen liege. Ebenso verhalte es sich da, wo am Rande von L a $n$ g e r h a $\mathrm{n}$ s schen Inseln Tubuli direkt an Zellstränge der Inseln anzustoßen scheinen. Wirkliche Übergänge von einer Zellart in die andere seien bisher von niemand glaubhaft nachgewiesen. - Gewiß ist es außerordentlich schwer, ja fast unmöglich, mit völliger Sicherheit , Utbergänge" zu beweisen, da wir ja nur aus den vorhandenen Bildern Rückschlüsse machen können, aber daß mit allergrößter Wahrscheinlichkeit Übergänge zwischen den beiden Zellarten im postfötalen Leben, wenigstens unter pathologischen Verhältnissen, vorkommen, das glaube ich doch aus den von mir gesehenen Bildern entnehmen zu können.

Wie oben erwähnt, sind die einzigen Zellarten im Pankreas, die stärkere Pyroninfärbung annehmen, die Tubulus- und Inselzellen, wobei die Farbe an kleine Körnchen gebunden erscheint. Ist der Reichtum dieser beiden Zellarten an färbbaren Elementen auch im allgemeinen ein deutlich verschiedener, so meine ich, deutet dieses färberische Verhalten doch schon eine nähere Verwandtschaft zwischen beiden Zellarten an, während bei dieser Färbung z. B. Gangepithelien und Tubuluszellen sich recht verschieden verhalten. Jedenfalls ist an und für sich die morphologische Verschiedenheit der beiden Zellarten nicht so groß, daß von vornherein Übergänge als unmöglich zu bezeichnen wären, besonders wenn man noch in Betracht zieht, daß die Kerne beider Zellarten äußerst ähnlich, wenn nicht völlig gleich sind.

Noch weniger kann für die Selbständigkeit der Langerhans schen Inseln aus der Anordnung der Z ellen geschlossen werden. Wenn behauptet wird, das Vorkommen von granulierten, von Tubuluszellen in einer sonst gut abgegrenzten $\mathrm{L}$ a $\mathrm{n}$ g e r h a $\mathrm{n}$ s schen. Insel sei einfach durch Zufälligkeit der Schnittrichtung bei den so häufig unregelmäßig gestalteten Inseln bedingt, so trifft das sicherlich nur für einen Teil der Beobachtungen zu. Wie sollte man sonst Bilder erklären können, wo mitten in den aus typischen Inselzellen gebildeten Zellsträngen einzeln oder zu mehreren Zellen liegen, die in jeder Beziehung den typischen Tubuluszellen gleichen. Gerade bei der Anwendung der MethylgrünPyroninfärbung kann man solche Befunde ziemlich häufig erheben, da die Tubuluszellen sich durch ihre intensiv rote Farbe deutlich abheben. Diese Zellen liegen oft völlig umschlossen von Inselzellen in den Zellsträngen, und si ch e r kann man hier das Dazwischenliegen auch feinster faseriger Elemente ausschließen. Wären diese Zellen Abschnitte eines zufällig durch die Schnittrichtung innerhalb einer Inselausbuchtung abgeschnittenen Tubulus, so müBte man da, wo mehrere solche Zellen zusammenliegen, doch wenigstens eine Andeutung einer drüsigen Anordnung der Zellen erwarten müssen, ein Befund, den man aber nur ganz ausnahmsweise erheben kann. Dabei sind die Zellen häufig völlig in ihrer Längsachse 
getroffen, nicht etwa nur tangential ihr basaler Abschnitt durch den Schnitt abgetrennt.

Sehr viel häufiger noch als die eben beschriebenen Bilder bekommt man solche zu Gesicht, wo am Rande einer im übrigen gut ausgebildeten Insel sich Ưbergänge zwischen den Balken der Inselzellen und d e n b e n a c h b a r te n Tu bu 1 i konstatieren lassen (siehe Tat. VII, Fig. 2 u. 3 ). Recht bemerkenswert erscheint es mir nun, daß die in näherem Zusammenhang mit den Inseln stehenden Tubuluszellen eine deutliche Anordnung zu drüsigen Gebilden recht häufig vermissen lassen und erst in einiger Entfernung von der Insel wieder eine deutliche drüsige Anordnung zeigen. An anderen Stellen wieder zeigen die an die Tubuli anstoßenden Inselzellen ihrerseits an Drüsen erinnernde Lagerung. Weiter sieht man in der Regel, daß an solchen Stellen die T u b u 1 u szellen ganz allmählieh den Charakter der Inselzellen a n n ehmen. Zuerst sieht man Tubuluszellen, die nicht mehr die typische Kegelform aufweisen und Zymogenkörnchen vermissen lassen. Ihre Form wird mehr rundlich, ihr Protoplasma zeigt aber noch starke Pyroninfärbung. Die darauf folgenden Zellen zeigen schon schwächere Rotfärbung, indem nur noch der basalste Teil des Leibes rot gefärbt ist, im übrigen Protoplasma aber nur noch einzelne rote Körnchen zu sehen sind. Dann folgen Zellen, die sich nur durch den reichlicheren Gehalt an roten Körnchen von den gewöhnlichen Inselzellen unterscheiden und schließlich ganz typische Inselzellen. Bei keiner anderen Färbung konnte ich diese Verhältnisse so deutlich sehen wie bei der Methylgrün-Pyroninfärbung, auch nicht bei der von $\mathrm{Karakascheff^{4 }}$ angewandten Färbung mit polychromem Methylenblau, obwohl auch sie ganz hübsche Bilder ergibt. Bei allen anderen. Färbungen ersèheint der Übergang von Tubulus- zu Inselzellen schroff, offenbar weil die Affinität dieser Farbstoffe zu den Körnchen im Zellprotoplasma nicht groß genug ist, um auch die vereinzelten Körnchen in den Inselzellen noch deutlich zur Anschauung zu bringen. Allerdings sieht man nicht an allen Stellen, wo Insel- und Tubuluszellen unmittelbar aneinander stoßen, solche Ủbergangsbilder schön ausgebildet. Vielleicht darf man hier annehmen, daß im Augenblick des Todes gerade ein stationäres Verhältnis bestand und der Übergang von Tubulus in Inselzellen, beziehungsweise der umgekehrte Vorgang ruhte. Jedenfalls findet man die oben geschilderten Bilder recht häufig, und es bleibt meiner Ansicht nach bei solchen Befunden nur die Annahme des Übergangs der einen Zellart in die andere übrig.

Nun ist von den Untersuchern, die in den $L$ a $n$ ger $h$ a $n$ schen Inseln völlig selbständige Gebilde erblicken, als Haupteinwand immer geltend gemacht worden, daß die Inseln von einer sie völlig umschließenden, bindegewebigen Kapsel umgeben seien. Weich sel ba u m ${ }^{5}$ will nur in den Fällen Lücken in den Kapseln oder völliges (?) Fehlen derselben zugeben, wo regeneratorische Vorgänge zur Vergrößerung der Inseln und dadurch za einex Art Sprengung der Kapsel 
geführt haben. Die sorgfältigen Untersuchungen besonders Herxheimers und seiner Schüler ${ }^{2}$ haben meines Erachtens den Beweis erbracht, daß diese Ansicht unhaltbar ist, und ich kann auf Grund meiner eigenen Untersuchungen auch nur konstatieren, daß außerordentlich häufig sich $\mathrm{kaum}$ ein e $\mathrm{An}$ d e utung einer Kapsel finden läBt. Bei den oben geschilderten, Ut̉bergangsbildern" ließ sich auch bei stärkster Vergrößerung keine Spur von Gewebe crkennen, das Insel- von Tubuluszellen trennte; $Z$ elle stö Bt un mittelb a $\mathrm{r}$ an $\mathrm{Z}$ elle. Auch wenn eine deutliche bindegewebige Hïlle die Inseln umgibt, kann das nicht als besonderes Charakteristikum der L ang e r h a n s schen Inseln aufgefaßt werden, denn auch das interlobuläre Bindegewebe umscheidet nicht selten kleinere, aus Tubuli bestehende Läppchen völlig.

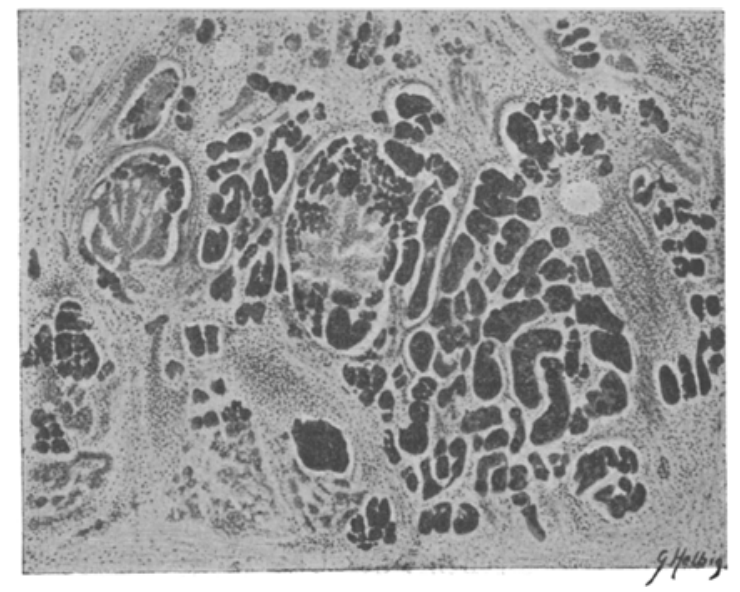

Fig. 1.

Noch anderes ist für die Selbständigkeit der Inseln geltend gemacht worden. So erklärte Helly ${ }^{6} 1909$ anläßlich eines Vortrags von Herxheimer: „Aus der Tatsache, daß pathologischerweise sich Kapseln nur um die Inseln bilden und innerhalb der Kapseln nicht Pankreas- und Inselgewebe vereinigt sind, geht hervor, daß ein gegenseitiger Ersatz nicht vorhanden ist." Auch diese Behauptung $\mathrm{H}$ elly s ist, ganz abgesehen von der Schlußfolgerung, nicht richtig. Schon 1906 schrieb Herxheimer ${ }^{2}$ : „Doch kann auch eine Zellinsel zugleich mit dem sich in sie umbildenden Pankreasgewebe von Bindegewebe umrahmt sein". Solche Befunde kann man in Bauchspeicheldrüsen, die aus diesen oder jenen Gründen ,zirrhotisch" verändert sind, recht häufig erheben (siehe Textfig. 1): Aber nicht nur in diesen Fällen, wo oft Inseln und übrige Parenchymreste nur noch in Lüeken des schwieligen, massigen Bindegewebes liegen, man von eigentlichen Kapseln also kaum noch reden kann, findet man solche Bilder. Auch in Fällen, wo das Pankreas keine oder nur höchst geringe Vermehrung des Bindegewebes 
zeigt, sieht man nicht gänz selten, Inselin, die teils arus Inselzellen, teils aus Tubuluszellen bestehen und von einer zarten, bindegewebigen Membran umhïllt und scharf abgegrenzt sind (siehe Taf. 떠, Fig. 4). Gerade diese Bilder sind es, die durchaus den Eindruck erwecken, daß die Langerhans schen Inseln keine einheitlichen, selbständigen Gebilde sind. Hinzu kommt, daß man auch Inseln findet oder richtiger Anhäufungen von Inselzellen, die in ihrer Form völlig von dem üblichen Bilde abweichen. Nicht mehr oder minder rundliche, scharf begrenzte Gebilde, die wenigstens teilweise durch faseriges Gewebe abgegrenzt sind, sieht man da, sondern längliche Zellzüge, die sich zwischen die Tubuli einschieben und keine Spur von einer „Kapsel“ erkennen lassen.

Alle diese Befunde lassen meiner Meinung nach nur die eine Erklärung zu, daß fließende Übergänge zwischen $\mathrm{L}$ a n g erh a ns schen Zellinseln und den Tubuli bestehen, und es würde sich nun wieder die Frage erheben: „Sind diese Befundeals Neubildung von Inseln aus dem übrigen Paren ch y $m$ aufzufassen, wie zahlreiche Untersucher annehmen, oder handelt es sich hier um den umgekehrten Prozeb, eine Bildung von Tubuluszellen aus Inselgewebe?"

Unzweifelhaft läßt sich die Mehrzahl der erwähnten Bilder nach beiden Richtungen hin erklären, und es dürfte unmöglich sein, mit Sicherheit nachzuweisen, da $\ddot{B}$ nur die eine Entstehungsweise denkbar ist. Ieh möchte mich darin völlig Her $x$ h e i m e ${ }^{2}$ ansehließen, wenn er sagt: „Eine Umwandlung von Drüsengewebe in Inseln halte ich . . . für bewiesen, eine Rückwandlung der Inseln in Drüsengewebe halte ich für theoretisch möglich, aber nicht für sicher nachgewiesen." Für die Umwandlung von Inseln in Drüsengewebe könnten vielleicht Befunde sprechen, wo Tubuluszellen ohne deutliche drüsige Anordnung sich in scheinbar deutlich abgegrenzten $\mathrm{L}$ angerhans schen Inseln finden und wo ein $\mathrm{Zu}$ sammenhang zwischen diesen Zellen und dem übrigen Parenchym sich nicht ohne weiteres nachweisen läßt. Für die gegenteilige Ansicht spricht aber meines Erachtens die Mehrzahl der Befunde. Hier wäre vor allen Dingen die of $\mathrm{t} \mathrm{un}$ geheuer grobe Anzahl von Langerhansschen Inseln in solchen Bauchspeicheldrüsen anzuführen, die durch pathologische Veränderungen bis auf kleine Reste des Drüsenparenchyms vernichtet sind. Wenn man eine Umbildung von Tubuluszellen aus Inseln annehmen will, warum sieht man dann gerade hier so außerordentlich häufig im schwieligen Bindegewebe oder im Fettgewebe Haufen von $\mathrm{L}$ a $\mathrm{n}$ g e r h a $\mathrm{n}$ s schen Inseln, ohne daß in ihrer Umgebung auch nur eine Andeutung von Tubuluszellen zu sehen wäre? Der Fortfall der Hauptmasse des sezernierenden Parenchyms sollte da doch einen großen Reiz auf die $\mathrm{L}$ a $\mathrm{n}$ ger $\mathrm{h}$ a $\mathrm{n}$ s schen Inseln in dieser Beziehung ausüben. Statt dessen zeigen aber auch die Inseln nicht selten an solchen Stellen, wo sie besonders eng von den pathologisch neugebildeten Geweben umschlossen sind, Veränderungen, die darin bestehen, daß sie die normal in ihrem Zelleib vorhandenen, mit Pyronin 
stark färbbaren Körnchen verlieren und nun als nur schwach diffus rosa gefärbte Elemente immer mehr den Epithelien der Ausführungsgänge ähnlich werden. An solchen Stellen konnte ich vereinzelt auch Befunde erheben, die den Schluß nahelegten, daß in der Peripherie dieser Inseln sich aus den Zellsträngen neue Drüsengänge bildeten, wie sie $\mathrm{Herxh}$ eime ${ }^{2}$ beschrieben hat. Nicht nur im Schwanzteil und im Körper des Pankreas können bei schweren Veränderungen des Organs solche Inselmassen entstehen, sondern auch im Kopf, wo die Inseln normalerweise anerkannt so viel seltener sind, kann man sie gelegentlich beobachten. So liegen sie in dem einen der von mir beobachteten Fälle in dem atrophisehen

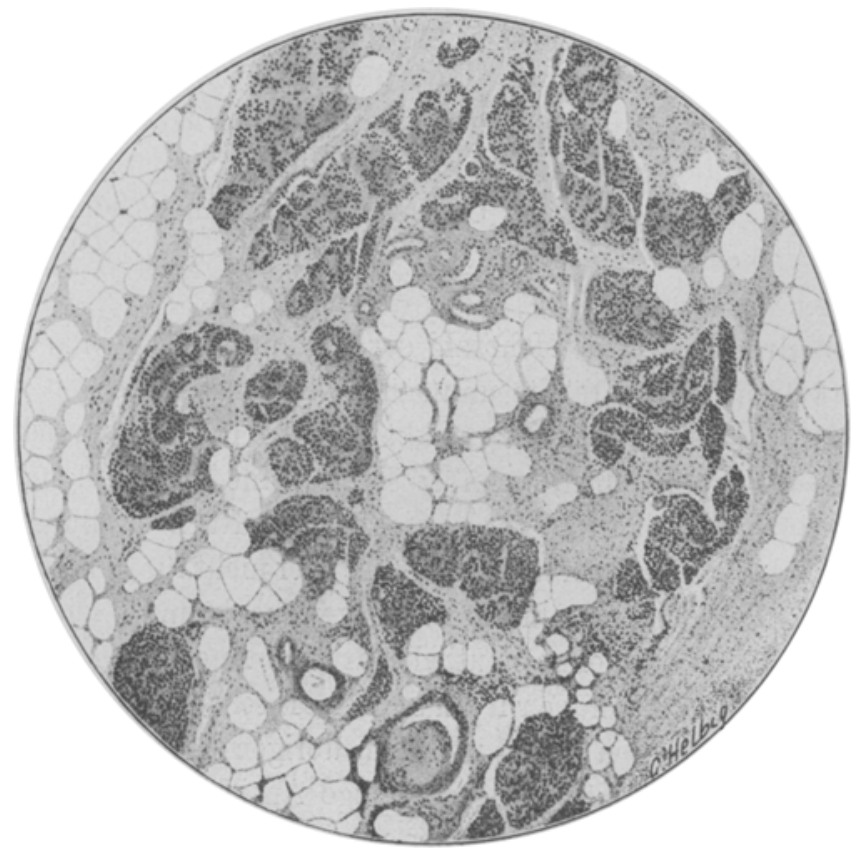

Fig. 2.

und fettdurchwachsenen Pankreas in dichtem Haufen im Kopfteil, während von Tubuli nichts, von Gängen nur sehr wenig zu sehen ist (Textfig. 2). Die Inselzellen zeigen nun mitunter, auch in Fällen, wo weder Diabetes noch Leberzirrhose bestand, auch an ihren Kernen Veränderungen, die nur als degenerative aufgefaßt werden können. Oft zeigt keine einzige der sicher stark vermehrten Inseln Zellen von normaler Beschaffenheit, so daß man doch wohl kaum annehmen darf, daß diese Inselvermehrung durch Regeneration von den Inseln selbst aus entstanden sei. Verfolgt man die Parenchymveränderungen bei schweren Pankreaserkrankungen, besonders bei ,Zirrhosen", so findet man an den besterhaltenen Stellen neben vereinzelten gut erhaltenen Tubuli solche, die in engem Zusammenhang mit, oft sehr 
kleinen, L a n g e r h a n s schen Inseln alle Übergänge ihrer Zellen zu den Inselzellen aufweisen, wobei ab und zu ein auffälliger, nicht immer ganz klarer Zusammenhang zwischen den Inselzellbalken und den ,zentroazinären" Zellen zu bestehen scheint. An anderen Stellen finden sich zahlreiche $\mathrm{L}$ a $\mathrm{n}$ g e r h a $\mathrm{n}$ s sche Inseln mit ganz spärlichen Resten von Tubuli in der nächsten Nachbarschaft und schließlich weiterhin nur noch $\mathrm{L}$ a $\mathrm{ng}$ e $\mathrm{rh}$ a $\mathrm{n}$ sche Inseln in die Norm weit übersteigender Zahl. Solche Befunde lassen, glaube ich, nur die Deutung zu, daß hier die L a $\mathrm{n}$ g e r h a $\mathrm{n}$ s schen Inseln aus den zugrunde gehenden Tubuli entstanden sind.

Nimmt man an, daß die Zellen der $\mathrm{L}$ a $\mathrm{n}$ g e $\mathrm{r}$ h a $\mathrm{n}$ s schen Inseln weniger hoch differenziert sind als die der Tubuli, wofür die histologischen Einzelheiten, wie sie gerade bei der Methylgrü-Pyroninfärbung hervortreten, in vieler Beziehung doch zu sprechen scheinen, so könnte man diese Vorgänge so auffassen, daß die am weitesten differenzierten Tubuluszellen sich, unter pathologischen Verhältnissen wenigstens, in die weniger h o e h differenzierten Inselepithelien und diese vielleicht sogar in die noch weniger differenzierten Epithelien der Ausführungsgänge z u r ü c k v e r w a n d e ln können. Eine solche Annahme hätte ein Analogon in der Umbildung von Leberzellbalken in Gallenkapillaren bei Leberzirrhose oder auch in der Umwandlung der hochdifferenzierten Epithelien der Tubuli contorti der Niere in kleine kubische Epithelien, wie man es bei Schrumpfnieren und ähnlichen Veränderungen findet. In allen diesen Fällen haben diese Zellen ihre frühere wichtige Funktion eingebüßt und, so glaube ich, liegt der Schluß nahe, daß es sich auch bei den Langerhansschen Inseln um nicht funktionierende bzw. funktionsuntüchtige Teile des Pankreas parench y m s handelt, eine Anschauung, die auch v o $n$. $\mathrm{H}$ a $n$ s e m a $n n^{3}$ erst neuerdings wieder verteidigt hat. Berücksichtigt man ferner, daß die Inselzellen bei Färbung mit Sudan III fast regelmäßig eine schwächere oder stärkere Rotfärbung zeigen, so daß sie sich hierdurch oft recht deutlich vom übrigen Parenchym abheben, so kann auch dieser Befund für die Anschauung verwertet werden, daß es sich um rückgebildete, degenerierende Zellen handelt. Wird andererseits die Möglichkeit zugegeben 7, daß in vereinzelten Fällen sich aus den Epithelien der Gallenkapillaren neue Leberzellen entwickeln können, so würde das der Annahme entsprechen, daß unter Umständen aus den weniger differenzierten Inselzellen die hochdifferenzierten Tubuluszellen entstehen können.

Ich komme daher zu folgenden Schlußfolgerungen.

1. Die Langerhansschen Inseln sind keine selbständigen Gebilde.

2. Sichere Übergänge zwischen Insel-und Tubulusz ellen sind häufig. 
3. Die Langerhansschen Inseln besitzenkeine eigentli e h e $\mathrm{Kapsel}$. Wo eine solche vorhanden zu sein scheint, hat sie nicht mehr Bedeutung als das übrige interlobuläre Bindegewebe.

4. Die Langerhansschen Inseln entstehen in der Hauptsache aus Tubuli; der umgekehrte Prozeß ist nicht völlig auszuschließen.

5. Die Langerhansschen Inseln sind als rückge • bildete, wahrscheinlich nicht funktionierende und funktionsuntüchtige Parenchymteile aufzufassen, ob sie als „Reservematerial“ in Frage kommen können, ist zweifelhaft.

Mit diesen Folgerungen finde ich mich in Übereinstimmung mit einer großen Zahl von Untersuchern des Pankreas. Bei der Veröffentlichung des Vorstehenden leitete mich einmal aber der Gedanke, daß tatsächlich durch die Anwendung der Methylgrün-Pyroninfärbung neue Befunde erhoben werden können, und weiter die Erwägung, daß bei der Wichtigkeit der Frage nach der Selbständigkeit der $\mathrm{L}$ a $\mathrm{n}$ gerhan s schen Inseln für die "Inseltheorie" des Diabetes neue Beobachtungen, die gegen die Selbständigkeit dieser Gebilde sprechen, von Bedeutung sind.

\section{Literatur.}

(Zusammenstellungen der sehr umfangreichen Literatur über Pankreas und Langer $\mathrm{h}$ a $\mathrm{n}$ s sche Inseln sind in letzter Zeit in so erschöpfender Weise (vgl. E. S a n e r b e ck, Die L ang e r h a $\mathrm{n}$ s schen Inseln des Pankreas und ihre Beziehung zum Diabetes mellitus; Ergebn. d. allg. Path. u. Path. Anat. 1902, II. Abt. und F. A l b r e c h t, Pathologie der Banchspeicheldrüse ; ebenda 1911, II. Abt. ) erfolgt, daß hier nur die im Text direkt erwähnten Veröffentlichungen angeführt sind.)

1. Pappenheim, Ztlbl. f. allg. Path. u. path. Anat. 1912. - 2. Herxheimer, Utber Pankreaszirrhose (bei Diabetes). Virch. Arch. Bd. 183. - D e r s el be Zur Pathologie des Pankreas. Verh. d. D. Path. Ges. 1909. - 3. v. H a n s e m a n n, Berl. klin. Wschr. 1912, Nr. 20. - D erselbe, Diskussion zum Herxheimerschen Vortrag. 1909. - 4. Kara$\mathrm{kasch}$ eff, Über das Verhalten der Langerhansschen Inseln bei "Diabetes mellitus. Verh. d. D. Path. Ges. 1904. - 5. W e i ch s el b a u m, Über Regeneration der Langerhansschen Inseln im menschlichen Pankreas. Sitzungsber. d. Kaiserl. Akad. d. Wissensch. in Wien 1908. - D e r s e l b e, Utber das Verhalten des Pankreas bei Diabetes mellitus. Ebenda 1910. D e r s elb e, Diskussion zum Herxheimersehen Vortrag 1909. - D e r s el b e und K y r le, Über das Verhalten der Langerhansschen Inseln des menschlichen Pankreas im fötalen und postfötalen Leben. Arch. f. mikrosk. Anat. u. Entwicklungsgesch. Bd. 74, 1909. - 6. H e 11 y, Diskussion zum Herxheimersehen Vortrag 1909. - 7. Sternberg, C., im Aschoffschen Lehrbuch Bd. 2, S. 259 .

\section{Erklärung der Abbildungen auf Taf. VII.}

Fig. 1 a. Scharf abgegrenzte L a n g e r ha n s sche Insel. Methylgrün-Pyroninfärbung. Leitz: Okul. 2, Obj. 6.

Fig. 1 b. Zwei Tubuluszellen und eine Inselzelle bei Betrachtung mit Ölimmersion.

Fig. 2. Tubuluszellen in $\mathrm{L}$ a $\mathrm{n}$ g e r h a $\mathrm{n} \mathrm{s}$ scher Insel. "Ubergangsbilder" zwischen diesen Zellen und den Inselzellen. Methylgrün-Pyroninfärbung. Okul. 2, Obj. 6. 


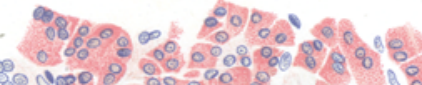

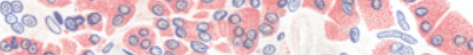
$0 \% 0 \%$ 198. $00.00 \%$

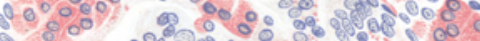
\%

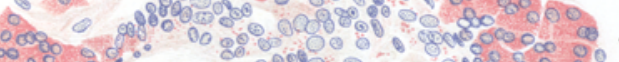
$96 \% \%$ \% $680^{\circ}$

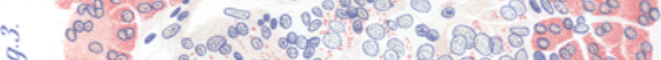

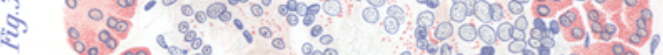

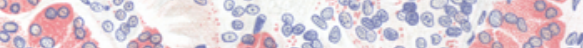

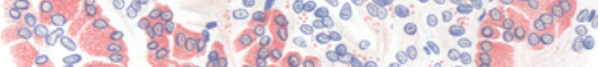

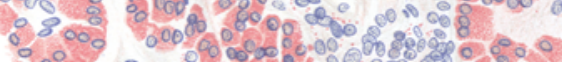

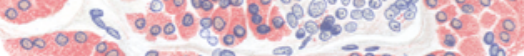

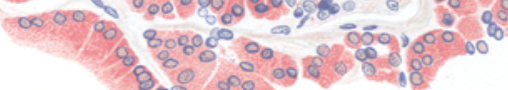
$30 \% 1000$
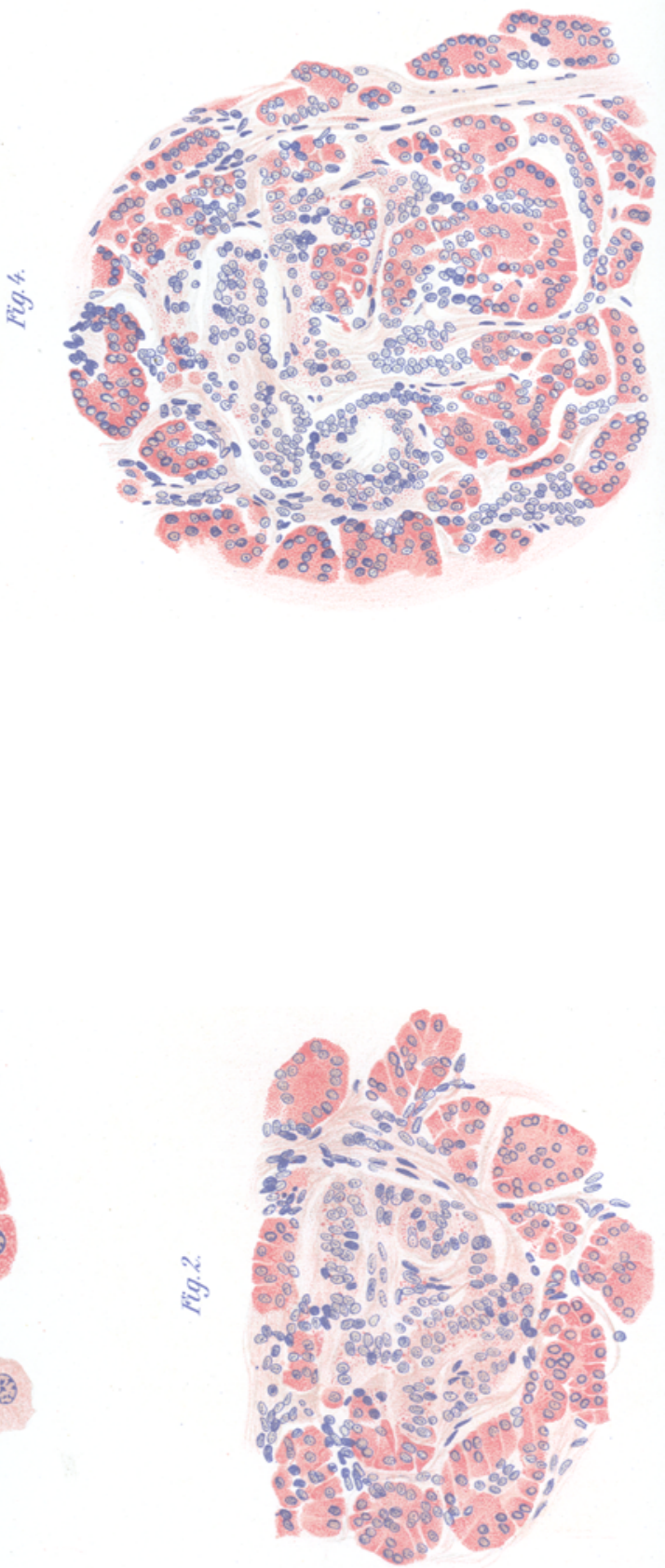

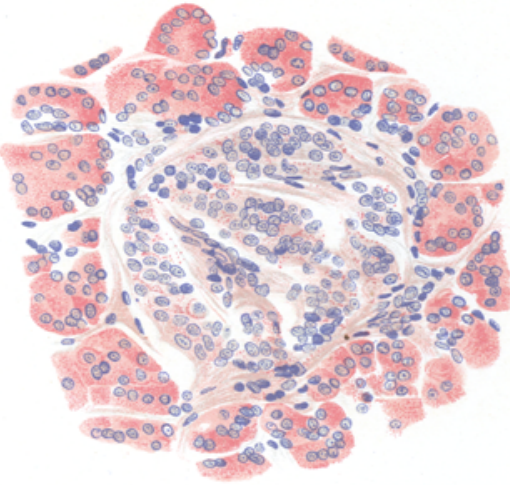

논 sic 
Fig. 3. Zahlreiche „Übergänge" zwischen Tubuli und L a nge r h a $\mathrm{n}$ s scher Insel. Methylgrün-Pyroninfärbung. Okul. 2, Obj. 6.

Fig. 4. "Insel" aus Insel- und Tubuluszellen fast gleichmäßig zusammengesetzt. MethylgrünPyroninfärbung. Okul. 1, Obj. 6 .

\section{XXI. \\ Über die pathologische Histologie der syphilitischen Aortitis mit besonderer Berücksichtigung des Vorkommens von Plasmazellen.}

(Aus dem Pathologischen Institut der Universität Berlin.)

Von

Dr. M. Fuku sh i (Tokio),

Volontärassistenten am Institut.

(Hierzu Taf. VIII.)

Während im Jahre 1885 die erste Arbeit D o e h les über die syphilitische Aortitis zunächst wenig Beachtung gefunden hatte, ist die Diskussion über die syphilitische Genese des von ihm beschriebenen Krankheitsbildes seit dem Kongreß in München im Jahre 1899 nicht verstummt, die Zahl seiner zunächst nur sehr vereinzelten Anhänger ständig gewachsen. Vor allem hatte sich auf diesem Kongreß $\mathrm{B}$ a u m garten gegen die Doehle-Heller sche Ansicht gewandt, indem er hervorhob, daß er bei einem großen, in Königsberg untersuchten Material niemals bei Paralytikern und Syphilitikern an der Aorta arteriitische Veränderungen gesehen habe, die er von der gewöhnlichen Arteriosklerose abgrenzen und als spezifisch syphilitisch hätte ansehen können. Nach $\mathrm{P}$ o n f i c $\mathrm{k}$ sollte der chronische Alkoholismus diese Veränderungen hervorbringen können, nach $\mathrm{Zi}$ e $\mathrm{g} l$ er sich das von D o e h l e beschriebene Krankheitsbild auch bei Infektionen mit Streptokokken und Staphylokokken finden. v. H. a s e m a n n behauptete, die Lokalisation des Prozesses im oberen Teil der Aorta sei kein Charakteristikum für die Spezifität der Erkrankung, da er im unteren Teil der Aorta ein zweifellos syphilitisches Aneurysma gesehen habe.

Standen ganz im Anfang nur B o lli ing er und B e ne ke auf D o e h les Seite, so schlossen sich ihm bald $\mathrm{Straub}, \mathrm{Backhaus,} \mathrm{Simon,} \mathrm{Moll}$ und I s e $\mathrm{n} \mathrm{b}$ er $\mathrm{g}$ an, und die um das Jahr 1903 herum zahlreich erscheinenden Arbeiten, die sich mit dieser Frage beschäftigten, nahmen höchstens eine abwartende oder zweifelnde Haltung an, wie das auch auf der Kasseler Naturforscherversammlung (1903) zum Ausdruck kam. Hier berichteten $\mathrm{Ch}$ i a r i und $\mathrm{B}$ e n d a 